\title{
ROBUST SCHEDULING OF BATCH PROCESSES IN UNCERTAIN CASES
}

\author{
Soon-Ki Heo, Hong-Rok Son and In-Beum Lee
}

Department of Chemical Engineering, Pohang University of Science \& Technology

San 31, Hyoja-Dong, Pohang, Kyungbuk, 790-784, Korea

\begin{abstract}
In this paper, a robust scheduling method is suggested in the optimization of batch plant with uncertainties considering not only expected value but also variance. Many papers treating scenario-based stochastic programming took expected values as objective functions. However, the meaning of expected value itself is sum of the probability of each scenario times each objective value. It implies nothing but currently calculated biggest value. It doesn't work when unexpected event happens. Therefore it is required to consider an additional criterion. I will choose variance and standard deviation of objective function. Copyright (C) 2005 IFAC
\end{abstract}

Keywords: Batch, Mathematical programming, Multiple-criterion, Optimization, Stochastic programming, Variance

\section{MOTIVATION}

Researches on multiproduct and multipurpose batch plants have been carried out for a few decades. Because the batch plants have their flexibility of processes and they are suitable for high-value-added products with short product life cycle. In mathematical approaches, there follow several assumptions by limitation of formulation and usage of solver program. Especially, certain process variables are fixed and determined in problem. Although before current computers and solver programs are developed solving a deterministic problem itself has an impact. Recently it is more important that how useful solutions are provided in real process. Therefore, a deterministic solution has much limitation. Active researches for solving with uncertain parameters, which is not deterministic but has certain distribution, are required in current trends.

Researches on robust scheduling are performed by Pinedo and Weiss (1987), De et al. (1992), Petkov and
Maranas (1997), Harding and Floudas (1997), Vin and Ierapetritou (2001), Subramanian et al. (2001) and so on. Pinedo and Weiss (1987), De et al. (1992) and Vin and Ierapetritou (2001) suggested a robust scheduling method considering expected value and variance of uncertain parameters of which distributions are known.

Schmidt and Grossmann (1996) tackled optimization of sequence of testing tasks when a pharmaceutical company or an agrochemical company developed a new product and made several samples, which is a representative example of stochastic programming problem. Lee et al.(2004) scheduled their testing tasks considering retest of the most expensive test. Jain and Grossmann (1999) took into account resource constraint when several projects were simultaneously processed and would make influence on other projects. Maravelias and Grossmann (2001) solved design and planning of same kind of stochastic problem. They formulated determinant variables of design and planning steps 
and solved large size of problem with Lagrangean Decomposition method.

Originally in formulation/solving steps, problems including uncertain parameters can be solved only when values of the parameters are given. A solution obtained by a set of given values of the parameters is called 'wait and see solution'. But this solution only is meaningless. So many researchers make use of expected value considering all scenarios of possible parameters.

Most previous researches handling scenario based stochastic problems took expected value as objective function of their problem. However, the meaning of expected value itself is sum of the probability of each scenario times each objective value. It implies nothing but currently calculated biggest value. It doesn't work when unexpected event happens. Therefore it is required to consider an additional criterion. In the context of scheduling, robustness can be defined as a measure of resilience of the scheduling objective to change in the face of parameter uncertainty and disruptive events. Scheduling is performed to satisfy variety of different objectives such as makespan minimization or maximization of profit or production. The most important determinant variable of scheduling problem is sequence. Briefly speaking, robust scheduling of stochastic problem is taken as to find a sequence that minimizes the influences by any events. Next sections follow illustrative examples, results and discussion for further works carefully.

\section{ILLUSTRATIVE EXAMPLES}

When solving the problem containing uncertain parameters we must know the distribution of each parameter, which could be obtained by reference or experimental data from the past operations. I would examine robust scheduling with a simple example with the system that manufactures three products of A, $\mathrm{B}$ and $\mathrm{C}$ through 4 equipment units multiproduct batch process. Processing times in equipment unit 1 and 2 are not deterministic due to the characteristics of products and process. The processing times are assumed to be distributed like Table 1 . The number of all the scenarios is $846(3 \times 3 \times 4 \times 4 \times 3 \times 2)$. MILP formulation is below. To avoid the confusion between 'objective value' and 'objective function' here, I would note 'objective value' to makespan, cost and profit, which is the interesting value. 'Objective function' would be generally defined to be minimized or maximized in the model, like expected makespan or something

Objective function:

$\sum_{s} P_{s} \cdot M S_{s}$

$$
\begin{aligned}
& \sum_{s} P_{s} \cdot M S_{s}+\left(\sum_{s} P_{s} \cdot M S_{s}^{2}-\sum_{s}\left(P_{s} \cdot M S_{s}\right)^{2}\right) \\
& \sum_{s} P_{s} \cdot M S_{s}+\sqrt{\sum_{s} P_{s} \cdot M S_{s}^{2}-\sum_{s}\left(P_{s} \cdot M S_{s}\right)^{2}}
\end{aligned}
$$

$P_{s}$ implies probability and $M S_{s}$ is makespan of scenario $s$ respectively. Then, equation (1) is the expected makespan based all the scenarios. Equations (2a) and (2b) mean the sum of expected value and its variance or its standard deviation.

Subject to:

$$
\begin{array}{ll}
\sum_{i} X_{k i}=1 & \forall k \\
\sum_{k} X_{k i}=1 & \forall i
\end{array}
$$

$X_{k i}$ is $0-1$ variable, which is valued one when product $k$ is produced in ith sequence. Product $k$ must be processed one of all sequences, which equation (3) implies. Equation (4) means that one of the products must be processed in each sequence.

In unit $j$ at $i$ th sequence, the completion time of $i$ th product $\left(C_{i j s}\right)$ must be greater than the completion time of directly previous product $\left(C_{i-1, j, s}\right)$ plus its processing time $\left(P T_{k j s}\right)$ for each scenario $s$.

$$
C_{i j s} \geq C_{i-1, j, s}+\sum_{k} P T_{k j s} \cdot X_{k i} \quad \forall i, j, s
$$

In unit $j$ after second, each ith product is processed after the task of the previous unit is completed.

$$
\begin{aligned}
& C_{i j s} \geq C_{i, j-1, s}+\sum_{k} P T_{k j s} \cdot X_{k i} \\
& M S_{s} \geq C_{i j s} \quad \forall i, j
\end{aligned}
$$

Makespan is the completion time of the last product.

Table 1. The distribution of processing times

\begin{tabular}{cc|cccc}
\hline Prod. & Unit & \multicolumn{5}{|c}{ Processing time / Probability } \\
\hline \multirow{4}{*}{$\mathrm{A}$} & 1 & $15 / 0.4$ & $16 / 0.1$ & $17 / 0.5$ & - \\
& 2 & $8 / 0.55$ & $9 / 0.25$ & $11 / 0.2$ & - \\
& 3 & 11 & - & - & - \\
& 4 & 18 & - & - & - \\
\hline \multirow{4}{*}{$\mathrm{B}$} & 1 & $14 / 0.05$ & $16 / 0.6$ & $18 / 0.05$ & $19 / 0.3$ \\
& 2 & $8 / 0.45$ & $10 / 0.35$ & $13 / 0.05$ & $15 / 0.15$ \\
& 3 & 12 & - & - & - \\
& 4 & 9 & - & - & - \\
\hline \multirow{4}{*}{$\mathrm{C}$} & 1 & $15 / 0.45$ & $17 / 0.3$ & $20 / 0.25$ & - \\
& 2 & $7 / 0.4$ & $8 / 0.6$ & - & - \\
& 3 & 17 & - & - & - \\
\hline
\end{tabular}

When the model has an objective function of expected makespan, the best sequence is $\mathrm{A}, \mathrm{C}$ and $\mathrm{B}$. Whereas, robust model found the sequence of $\mathrm{B}, \mathrm{A}$ and C. A representative Gantt chart is shown in Figure 1. There are three diagrams in Figure 2. Figure 2(a) is the result when expected makespan, i.e. equation (1) is taken as the objective function. Figure 2(b) represents the distribution when equation (2) is used. 
And Figure 2(c) is built by equation (3) as objective function. The distributions of makespans are different from each other.

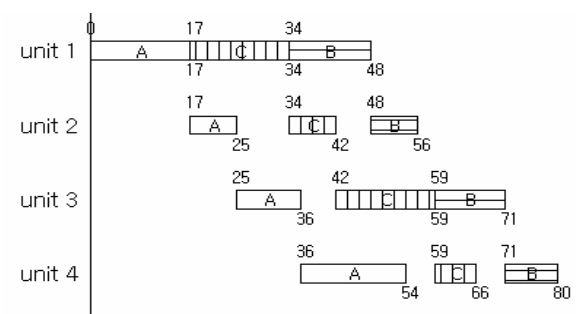

(a) Expected makespan

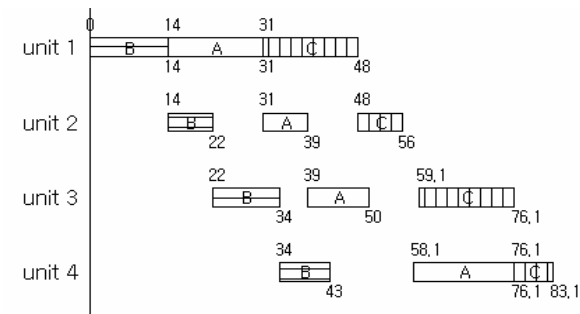

(b) Expected makespan plus variance

Figure 1. Gantt chart of example 1

In Figure 2, which is the best? Which of the three gives more exact answer "when is whole the production process over?" or "By when I could receive my order?" If Figure 2(a) is called 'normal', Figure 2(b) could result in 'lateness' but could give better answer of 'when'. Figure 2(c) is 'intermediate'. Expected values and variances are represented in Table 2. There is a tradeoff between fast completion and reliability. That implies which is important between the target value and the robustness. So alternative objective functions could be recommended.

$$
\begin{gathered}
(1-\lambda) \cdot \sum_{s} P_{s} \cdot M S_{s}+\lambda \cdot \sqrt{\sum_{s} P_{s} \cdot M S_{s}^{2}-\sum_{s}\left(P_{s} \cdot M S_{s}\right)^{2}} \\
0 \leq \lambda \leq 1 \quad \text { (2c) } \\
(1-\lambda) \cdot \sum_{s} P_{s} \cdot M S_{s}+\lambda \cdot\left(\sum_{s} P_{s} \cdot M S_{s}^{2}-\sum_{s}\left(P_{s} \cdot M S_{s}\right)^{2}\right) \\
0 \leq \lambda \leq 1
\end{gathered}
$$

If the value of $\lambda$ is 0 , this function is same with equation (1). If $\lambda$ is larger but smaller than 1 , it is getting narrower but could be shift to right. If the value of $\lambda$ is smaller but larger than 0 , the distribution of objective values is declined to shift to left but is getting broader. The value of $\lambda$ depends on decision makers. Published papers tackling stochastic problems with uncertain parameters almost took only expected values of cost or profit etc. as objective function. However additional criterion is suggested here in order to consider both objective value and its robustness.

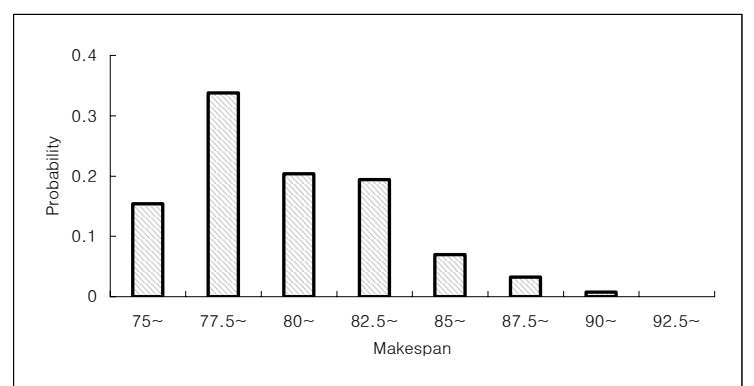

(a) Expected makespan

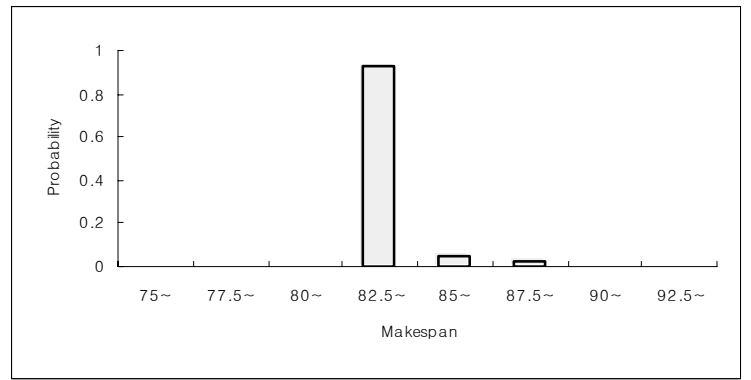

(b) Expected makespan plus variance

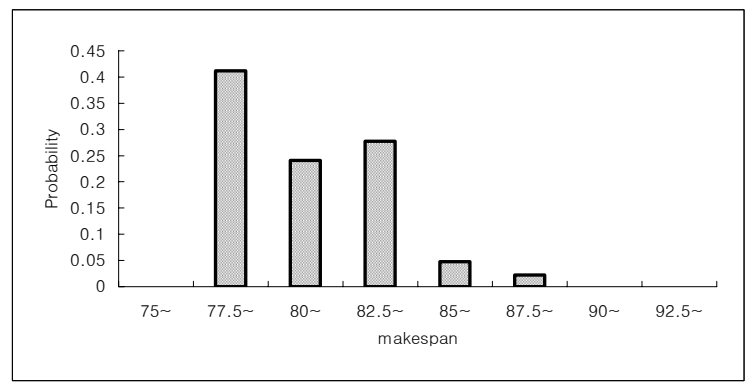

(c) Expected makespan plus standard deviation

Figure 2. Distributions of makespan with different objective function, example 1

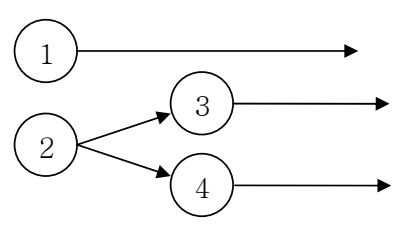

(a) slight robustness

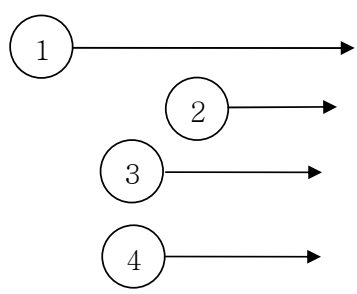

(b) heavy robustness

Figure 3. Robust schedules of testing tasks 
Now we bring another example which was treated by Schmidt and Grossmann(1996) to schedule testing tasks that must be passed to develop and issue a new pharmaceutical product. The parameter was given in Table 2 of their work. The formulation with the expected objective value brought the schedule like Figure 3(a). However, robust schedule, i.e. the value of $\lambda$ over 0.75 , found the schedule like Figure 3(b). During the scheduling to be robust, expected cost increased about $12.6 \%$. On the other hand, standard deviation reduced about 95\%. Which is more important factor between two criteria? Expected cost implies the sum of cost times of probability of each scenario. If the project is undergoing so well, all the tasks have to be performed. Then the word 'expected' is not so significant meaning. Instead, simultaneous start of all tasks is not so good policy, either. The recommended policy is that the longest task is undergone first and the others are done before the half.

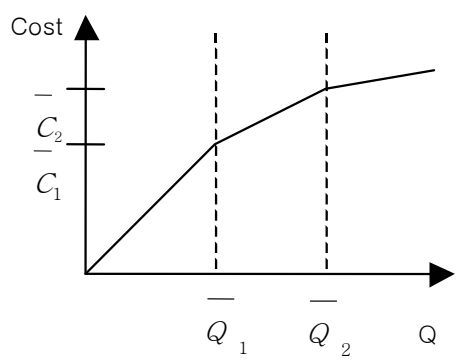

Figure 4. Quantity discount model

Heo et al.(2003) solved an integrated production and distribution of a chemical company who operates PVC plants in the view point of SCM. In the formulation quantity discount model was applied. The equations are below and referable figure is in Figure 4.

$$
\begin{aligned}
& Z_{r}=\left\{\begin{array}{l}
1, \text { if } Q \in\left[\bar{Q}_{r-1}, \bar{Q}_{r}\right] \\
0, \text { otherwise }
\end{array}\right. \\
& \bar{Q}_{r-1} Z_{r} \leq Q_{r} \leq \bar{Q}_{r} Z_{r} \\
& \sum_{r=1}^{N R} Z_{r}=1 \\
& Q=\sum_{r=1}^{N R} Q_{r}
\end{aligned}
$$

When they purchase the amount of $Q$, it is includes in only one interval of set ' $r$ ' by the equations $8-11$. The total profit is given in equation 12 .

$$
C=\sum_{r=1}^{N R}\left[\bar{C}_{r-1} Z_{r}+\left(Q_{r}-\bar{Q}_{r-1} Z_{r}\right) \frac{\bar{C}_{r}-\bar{C}_{r-1}}{\bar{Q}_{r}-\bar{Q}_{r-1}}\right]
$$

There are three suppliers of ethylene which is raw material of PVC, four plants and eleven warehouses in Korea. There are two suppliers, two plants and four warehouses respectively in China. They assumed that all the parameters were deterministic. However, we built a scenario of crude oil prices which was analyzed by data through last three years and has average value of the last year. Figure 5 represents the scenario. This scenario cannot provide the exact trends of the variable price, but gives the information how much probability it has had up to last three years.

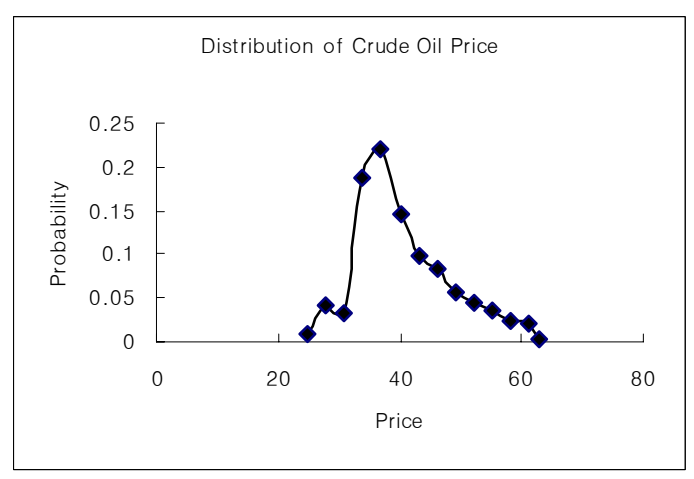

Figure 5. Scenario of Crude oil

This assumption will be applied when the manager has to build a contract of the whole year at the start point. The objective value is to maximize sales profit minus transportation, inventory and operating cost. This robust planning will make the profit, transportation and operating cost efficient against unexpectedly variable price of the crude oil and resources. As a result, 'robustness' bring different interval of ' $Q$ ' from plants to warehouses. For example, Ulsan plant transport different amount of PVC to warehouses under normal and robust planning. Refer to Figure 6. Robust planning tells Ulsan plant to transport more amounts to Ulsan warehouse to prepare for fluctuation of the crude oil price which could make an influence of resource, product prices and transportation, operation costs.

\section{DISCUSSION}

The application of proposed robust scheduling of stochastic programming with uncertainty method to other uncertain parameter seems to be reasonable. First of all, it is desirable to optimize the sequence of testing tasks when a pharmaceutical company or an agrochemical company developed a new product and made several samples, which were tackled by Schmidt and Grossmann (1996), Jain and Grossmann (1999) and Maravelias and Grossmann (2001). They used objective function with only expected value. 
Second, the price of crude oil is inclined to unexpectedly fluctuate. The proposed policy could not tell what the price of next day is, but could make the effect minimized. Third, a manager could estimate the budget easily due to the robust scheduling and preparation

Treating skill of MINLP problem has much to be improved. Although nonlinear terms of example 2 can be piecewise linearized, it could be impossible due to the characteristics of objective variables. Then, Generalized Benders Decomposition method could be appropriate.

\section{REFERENCES}

De, Prabuddha, Jay B. Ghosh and Charles E. Wells (1992). Expectation-variance analysis of job sequences under processing time uncertainty. International Journal of Production Economics, 28, 289-297.

Harding, S. T. and C. A. Floudas (1997). Global Optimization in Multiproduct and Multipurpose Batch Design under Uncertainty. Ind. Eng. Chem. Res. 36, 1644-1664.

Heo, Soon-Ki, Hong-Rok Son, Kyu-Hwang Lee, HoKyoung Lee, In-Beum Lee and Euy-Soo Lee (2003). Production and Distribution of Polyvinyl Chloride Considering Demands of Warehouses. Presented at Process Systems Engineering 2003, Kunming, China.

Jain, Vipul and Ignacio E. Grossmann (1999). Resource-Constrained Scheduling of Tests in New Product Development. Ind. Eng. Chem. Res. 38, 3013-3026.

Lee, Jong-Min, Soon-Ki Heo and In-Beum Lee (2004). Scheduling of Tests in New Product Development. Presented at 2004 KIChE Fall Meeting, Hoseo University, Korea.

Maravelias, Christos T. and Ignacio E. Grossmann (2001). Simultaneous Planning for New Product Development and Batch Manufacturing Facilities. Ind. Eng. Chem. Res. 40, 6147-6164.

Petkov, Spas B. and Costas D. Maranas (1997). Multiperiod Planning and Scheduling of Multiproduct Batch Plants under Demand Uncertainty. Ind. Eng. Chem. Res. 36, 4864-4881.

Pinedo, Michael and Gideon Weiss (1987). The "Largest Variance First" Policy in Some Stochastic Scheduling Problems. Operations Research, 35, 884-891.

Schmidt, Craig W. and Ignacio E. Grossmann (1996). Optimization Models for the Scheduling of Testing Tasks in New Product Development. Ind. Eng. Chem. Res. 35, 3498-3510.

Subrahmanyam, Sriram, Joseph F. Pekny and
Gintaras V. Reklaitis (1994). Design of Batch Chemical Plants under Market Uncertainty. Ind. Eng. Chem. Res. 33, 2688-2701.

Vin, Jeetmanyu P. and Marianthi G. Ierapetritou (2001). Robust Short-Term Scheduling of Multiproduct Batch Plants under Demand Uncertainty. Ind. Eng. Chem. Res. 40, 4543-4554.

Table 2 . The values of objective function and $\underline{\text { metric }}$

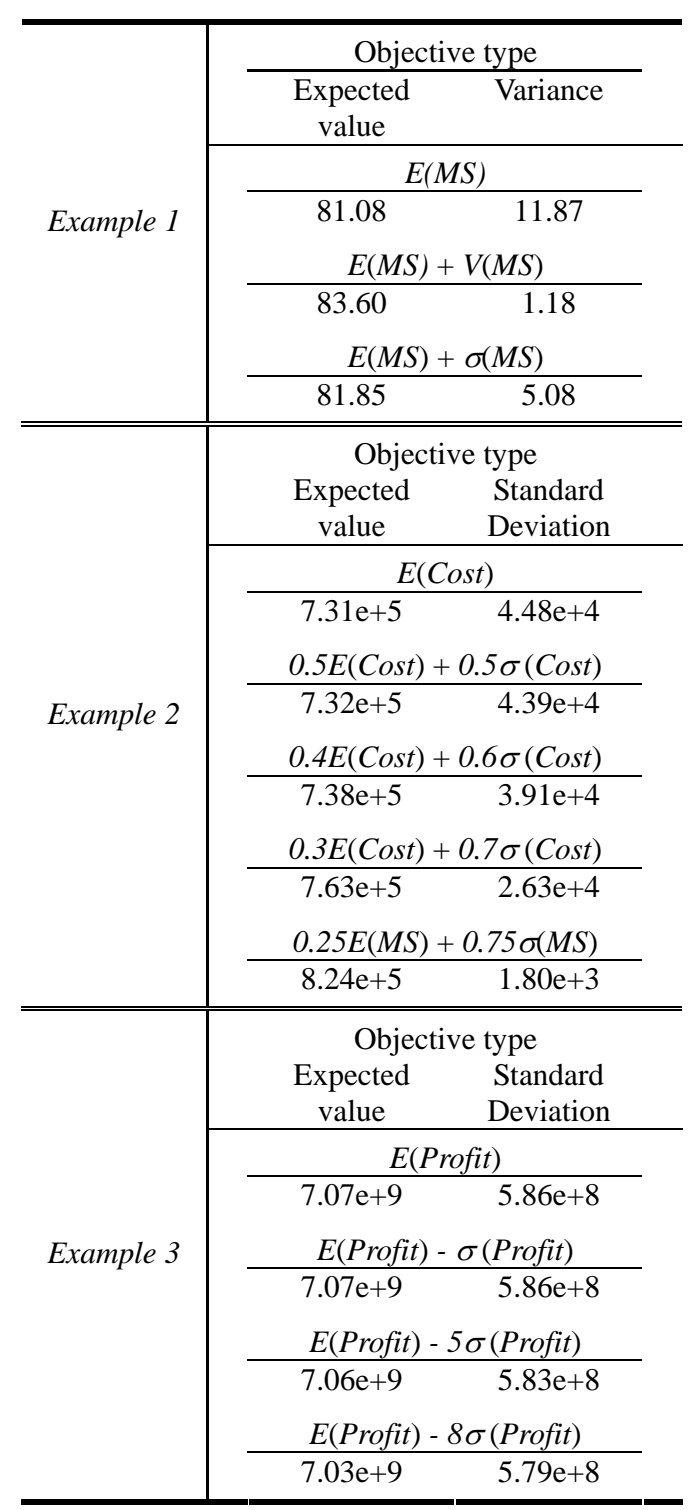

\section{NOMENCLATURES}

Indices

$i$ : ith sequence 
$j$ : unit

$k$ : product

$r$ : discrete point for quantity discount

$s$ : scenario

\section{Parameters}

$\lambda$ : weight of robustness

$\bar{C}_{r}$ : discrete price for quantity discount

$P_{s}$ : probability of scenario $s$

$\mathrm{PT}_{\mathrm{kjs}}$ : processing time of product $p$ in unit $j$ of scenario $s$

$\bar{Q}_{r}$ : discrete amount for quantity discount
$C:$ cost

$C_{i j s}$ : completion time of $i$ th product in unit $j$ of scenario $s$

$M S_{s}$ : makespan of scenario $s$

$Q$ : quantity

$X_{k i}$ : binary, 1 when product $k$ is processed in ith sequence

$Z_{r}$ : binary, 1 when $Q_{r}$ is in the interval $r-1$ and $r$

Variables

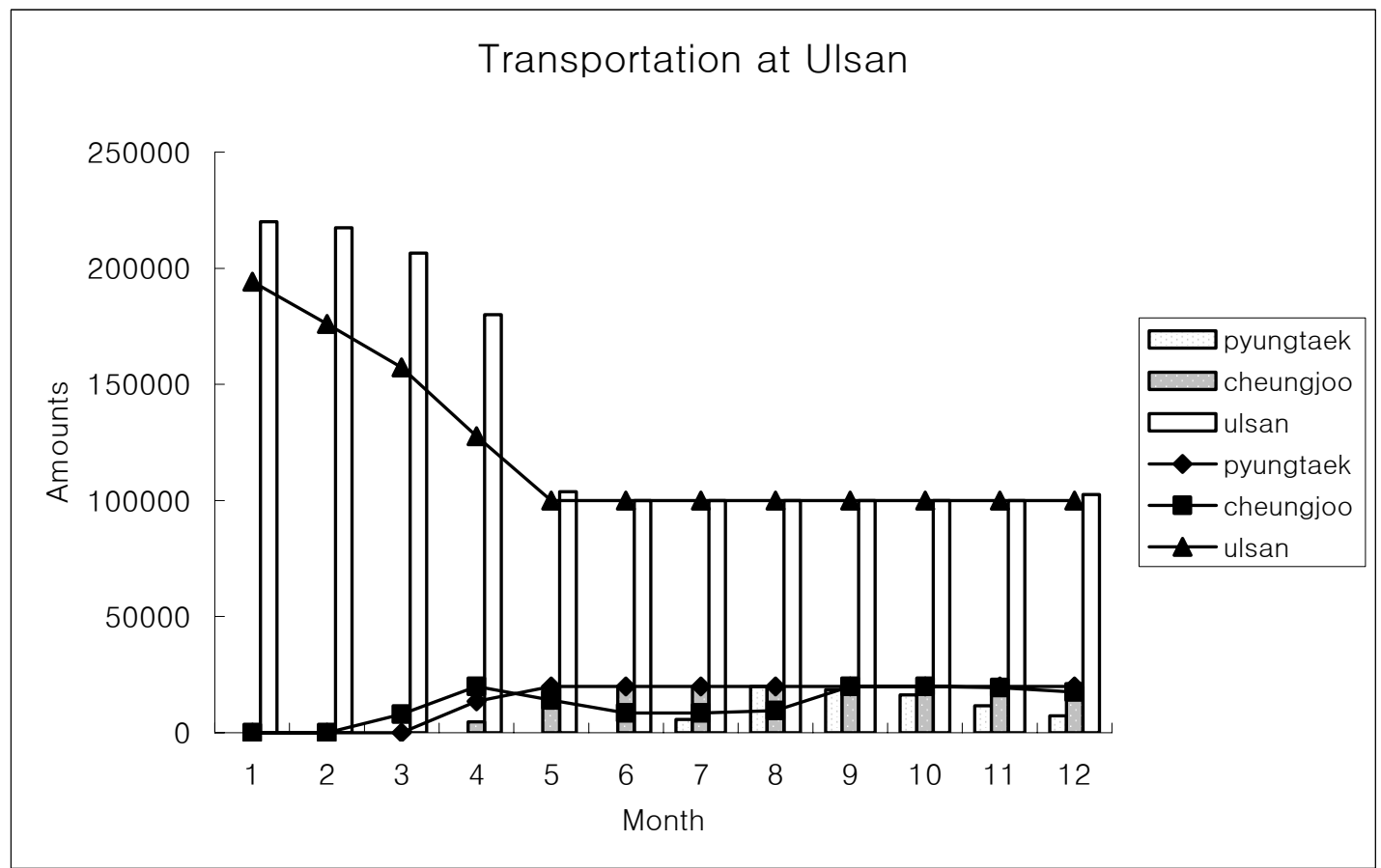

Figure 6. Comparison of Ulsan Plant (stick: robust, line and legend: normal) 